\title{
Time dependence of the proton and helium fluxes measured by PAMELA during solar minimum (2006 - 2009)
}

\author{
Nadir Marcelli* \\ INFN, Sezione di Roma "Tor Vergata", I-00133 Rome, Italy \\ Universita' degli studi di Roma "Tor Vergata", Rome, Italy \\ E-mail: nadir.marcelli@roma2.infn.it \\ M. Boezio \\ INFN, Sezione di Trieste, I-34149 Trieste, Italy \\ IFPU - Institute for Fundamental Physics of the Universe, Trieste, Italy \\ E-mail: mirko.boeziodts.infn.it
}

\section{A. Lenni}

INFN, Sezione di Trieste, I-34149 Trieste, Italy

IFPU - Institute for Fundamental Physics of the Universe, Trieste, Italy

Universita' degli studi di Trieste, Trieste, Italy

E-mail: alex.lenni@ts.infn.it

\section{Martucci}

INFN, Sezione di Roma “Tor Vergata”, I-00133 Rome, Italy

E-mail: matteo.martuccieroma2.infn.it

\section{W. Menn}

Universitat Siegen, Department of Physics, D-57068 Siegen, Germany

E-mail: menn@pamela.physik.uni-siegen.de

\section{R. Munini}

INFN, Sezione di Trieste, I-34149 Trieste, Italy

IFPU - Institute for Fundamental Physics of the Universe, Trieste, Italy

E-mail: riccardo.munini@ts.infn.it

\section{R. Sparvoli}

INFN, Sezione di Roma "Tor Vergata”, I-00133 Rome, Italy

Universita' degli studi di Roma "Tor Vergata”, Rome, Italy

E-mail: roberta.sparvolieroma2.infn.it

\section{O. P. M. Aslam}

North-West University, Centre for Space Research, 2520 Potchefstroom, South Africa

E-mail: aslamklr2003@gmail.com

\section{Bisschoff}

North-West University, Centre for Space Research, 2520 Potchefstroom, South Africa E-mail: driaanbegmail.com

\section{S. Potgieter}


North-West University, Centre for Space Research, 2520 Potchefstroom, South Africa

E-mail: marius.potgieter@nwu.ac.za

\section{On behalf of the PAMELA collaboration}

Precision measurements of the $\mathrm{Z}=2$ component in the cosmic radiation provide crucial information about both the origin and propagation of the helium nuclei in cosmic rays inside the Galaxy. Moreover, the precise measurement of the time-dependent helium nuclei fluxes allows an in-depth study of the solar modulation of such nuclei. These results can be compared with the widely studied modulation of protons, to possibly spot dependencies on particle's charge, mass or local interstellar spectra shape. The helium differential fluxes together with comparison between helium and proton energy spectra, measured by the PAMELA experiment down to $80 \mathrm{MeV}$, has been studied in the unusually long minimum of solar cycle 23 (July 2006 December 2009) over a 1-month time basis.

36th International Cosmic Ray Conference -ICRC2019-

July 24th - August 1st, 2019

Madison, WI, U.S.A.

${ }^{*}$ Speaker. 


\section{Introduction}

Protons and helium are the most abundant species in cosmic rays, together they represent about $99 \%$ of the total flux. Measurements of their absolute fluxes and spectral shapes are extremely important for better understanding both the origin and propagation mechanisms of galactic cosmic rays (GCRs). Moreover, both proton and helium fluxes are essential ingredients to estimate the spectra of secondary particles resulting from interaction of GCRs with the interstellar medium (ISM). The propagation of CR in the galaxy modify their spectra and composition. Before reaching the Earth, cosmic rays particles enter the heliosphere and they are affected by the heliospheric magnetic field (HMF) transported by the expanding solar wind from the Sun to the outermost regions of the heliosphere. The interaction of these particles with HMF changes significantly the intensity profile of energy spectra below $\sim 30 \mathrm{GeV}$ for protons and $\sim 15 \mathrm{GeV} / n$ for helium with respect to the Local Interstellar Spectrum (LIS), i.e. the spectrum which would be measured outside the heliospheric boundaries. The magnitude of the intensity attenuation depends on the solar activity; this effect is known as solar modulation, e.g. see [Potgieter(2013), Strauss \& Potgieter(2014)]. Solar activity varies strongly with time, rising from a minimum level when the Sun is quiet (with cosmic rays then having a maximum intensity at Earth) to a maximum period (when cosmic rays have a minimum intensity at Earth) and then returning to a new minimum repeating the cycle. This solar cycle has a periodicity of approximately 11 years. The unusually long minimum activity of solar cycle $23^{\text {rd }}$ form 2006 to late 2009 (e.g. see [Mewaldt et al.(2010)]) caused an ideal low modulation conditions for GCRs and this is translated into a perfect environment to study the various processes that affect particles inside the heliosphere. After 2009, the solar activity increased again reaching its maximum in January 2014; the $24^{\text {th }}$ solar cycle maximum was classified as one of the weakest in recent years, e.g. see [Schröder et al.(2017), Aslam \& Badruddin(2015), Jiang et al.(2015)]. The PAMELA experiment collected data for almost 10 years and the instrument was well suited to measure the effect of solar modulation on many cosmic rays species, because of its redundant detectors and high precision measurements which permitted unprecedented statistics. The PAMELA collaboration published several papers on CR solar modulation: protons [Adriani et al.(2013)], electrons [Adriani et al.(2015)], the $e^{-} / e^{+}$ratio [Adriani et al.(2016)], hydrogen and helium isotopes [Munini et al., 2019] and soon total helium $\left({ }^{3} \mathrm{He}+{ }^{4} \mathrm{He}\right)$.

\section{The instrument}

PAMELA (A Payload for Antimatter-Matter Exploration and Light-nuclei Astrophysics) is a satellite-borne experiment designed to make long duration observations of the cosmic radiation from Low Earth Orbit [Picozza et al.(2007)]. The instrument collected GCRs for almost 10 years from 2006 June 15 when it was launched from the Baikonur cosmodrome in Kazakhstan, until January of 2016. The PAMELA instrument was hosted on board of the Russian satellite DK1 that orbited Earth at an altitude ranging between 350 and $610 \mathrm{~km}$ with an inclination of $70^{\circ}$. After 2010 the orbit was changed to a circular one at a constant altitude of about $500 \mathrm{~km}$.

The payload comprises a number of highly redundant detectors capable of identifying particles by providing charge, rigidity and velocity measurements over a very wide energy range. Multiple 


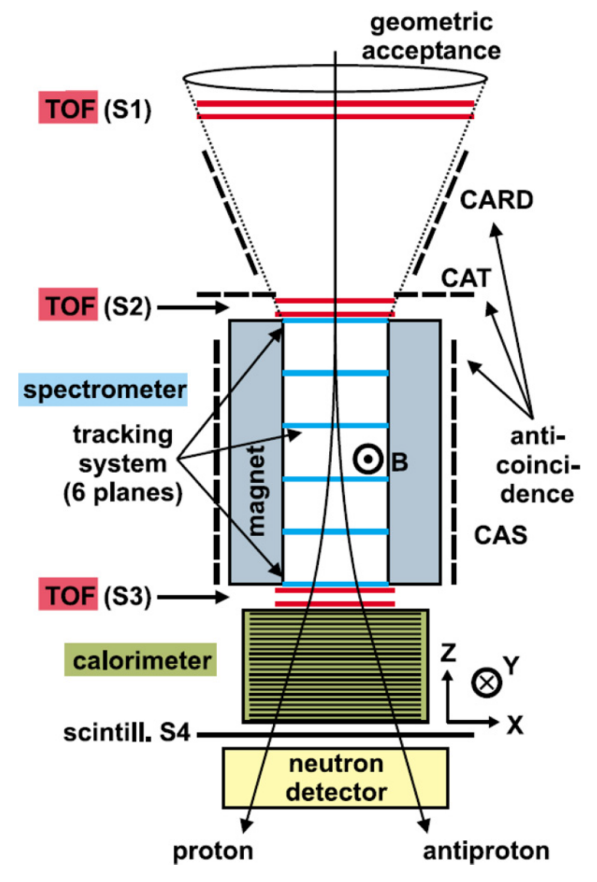

Figure 1: PAMELA and its sub-detectors.

sub-detectors are arranged around a magnetic spectrometer, composed of a silicon tracking system [Adriani et al.] placed inside a $0.43 \mathrm{~T}$ permanent magnet. The $300 \mathrm{~mm}$ thick double-sided Silicon sensors of the tracking system measure two independent impact coordinates on each plane, reconstructing with high accuracy the particle deflection, with a maximum detectable rigidity of $\sim 1.2 \mathrm{TV}$, and the sign of the electric charge $Z$. A system of six layers of plastic scintillators, arranged in three double planes (S1, S2, and S3), provides a fast trigger to acquire data. It contributes to particle identification measuring the ionization energy loss and the time of flight (ToF) of particles passing through with a resolution of $\sim 300 \mathrm{ps}$; this assures the determination of the absolute value of the particle charge plus allowing albedo particles rejection [Osteria et al.(2004)]. An electromagnetic imaging W/Si calorimeter (16.3 radiation lengths and 0.6 interaction lengths deep) provides hadron-lepton discrimination [Boezio et al.(2002)]. A neutron counter [Stozhkov et al.(2005)] contributes to the discrimination power by detecting the increased neutron production in the calorimeter associated with hadronic showers compared to electromagnetic ones, while a plastic scintillator, placed beneath the calorimeter, increases the identification of high-energy electrons. The whole apparatus is surrounded by an anti-coincidence system (AC) of three scintillators (CARD, CAS, and CAT) for the rejection of background events [Orsi et al.(2005)]. A comprehensive description of the instrument can be found in [Adriani et al.(2014)]. The payload is schematically shown in Figure 1.

\section{Analysis}

\subsection{Event Selection}

A clean helium sample was obtained applying several selection cuts to the data provided by 


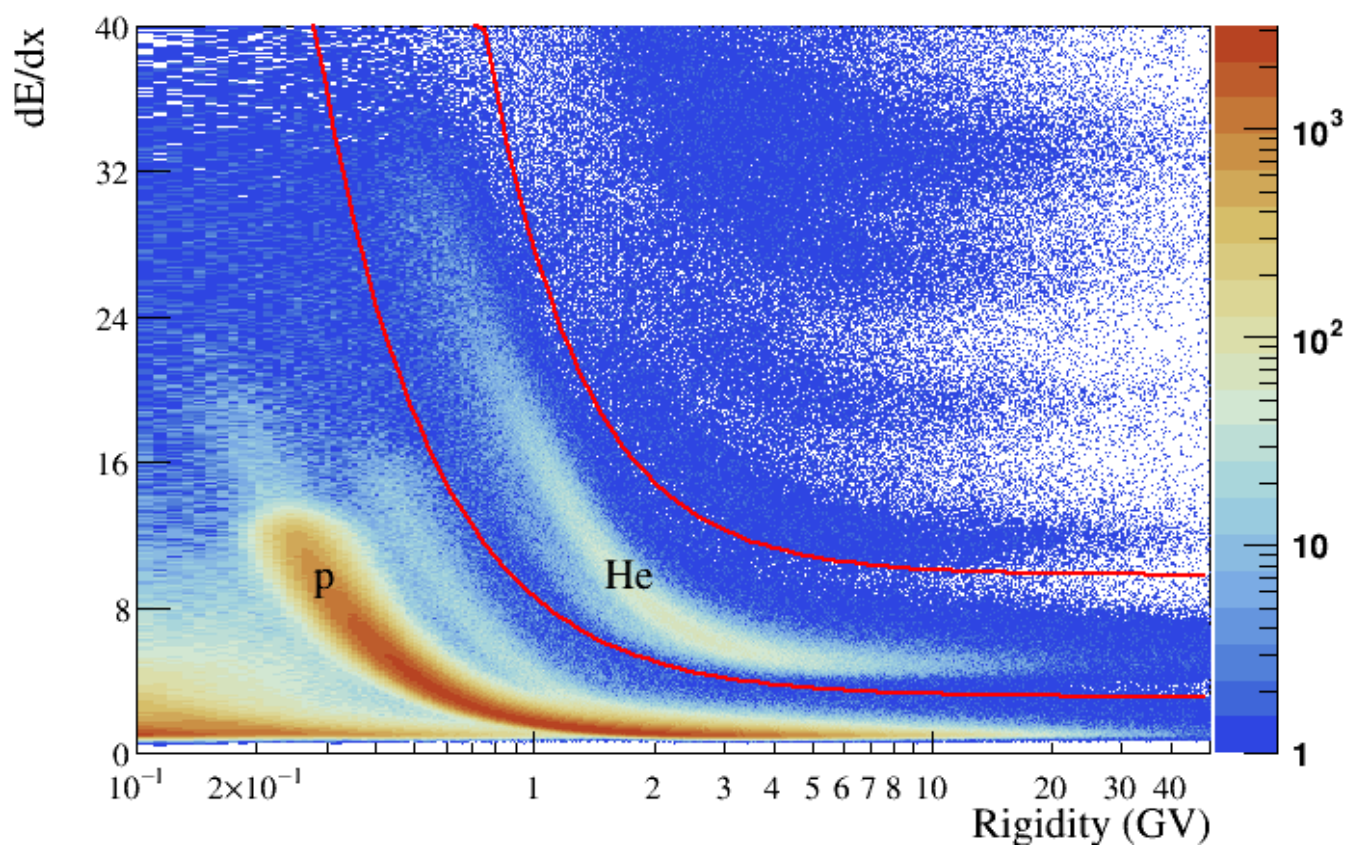

Figure 2: Energy loss distribution in the tracking system as a function of particle rigidity. The two red curves define the helium nuclei band which is clearly separated from the one of protons.

the PAMELA sub-detectors.

Helium candidates are selected requiring to have a single track fitted within the spectrometer fiducial volume where the reconstructed track is at least $1.5 \mathrm{~mm}$ away from the magnet walls. This sample must have a lever-arm of at least 4 silicon planes and a minimum of 3 hits on both the bending $x$-view and non-bending $y$-view. Only events with a good $\chi^{2}$ of the fitting algorithm are accepted. Albedo particle rejection is obtained requiring a positive $\beta=\frac{v}{c}$ measured by the ToF system; where $v$ is the velocity of the incoming particle and $c$ is the speed of light. Multi-particles event, produced from the interaction of primary helium interacting with the materials above the instruments, are rejected requiring no more than 1 hit paddle on each S1 and S2 planes and that those hits are associated to the extrapolated track from the spectrometer. Finally, helium nuclei are selected according to their mean energy release over the silicon planes of the tracking system. Only events which are inside the band defined by the two red curves in Figure 2 are selected.

\subsection{Flux estimation}

The absolute helium flux $\Phi(E)$ in kinetic energy was obtained as follows:

$$
\Phi(E)=\frac{N(E)}{G(E) \times L T \times \varepsilon(E) \times \Delta E}
$$

where $N(E)$ is the unfolded count distribution, $\varepsilon(E)$ the product of the single selections efficiencies, $G(E)$ the geometrical factor, $L T$ the live-time and $\Delta E$ the width of the energy interval. 


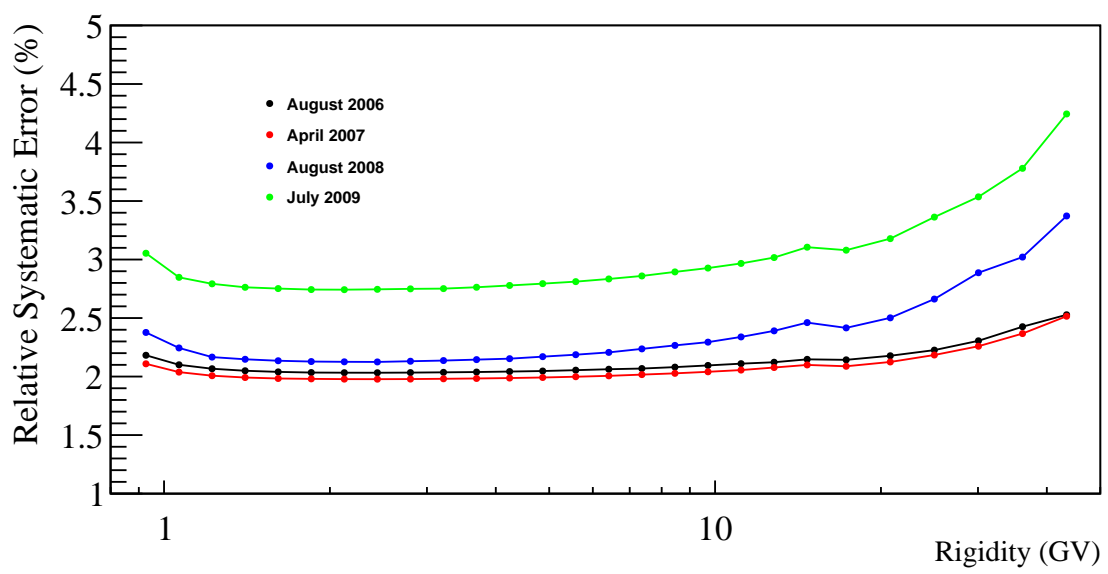

Figure 3: Relative systematic error as a function of the rigidity for four time intervals.

The redundant measurements provided by the PAMELA sub-detectors allows to evaluate selection efficiencies with flight data. When possible, the selection efficiencies were evaluated with flight data, while for the tracker selections efficiency Monte Carlo simulations were employed. In order to avoid any possible time variation of the detector response, the efficiencies were evaluated for each Carrington rotation.

Because of the finite spectrometer resolution and ionization energy losses, the helium rigidity measured with the tracker system differs from the initial energies at the top of the payload; to overcome these effects a Monte Carlo based unfolding procedure was performed, e.g. see [D'Agostini, 1995].

Finally, in order to correct for possible unaccounted time-dependent variations of the fluxes, each set of monthly-based fluxes was normalized at high energy $(30-50 \mathrm{GeV})$ to the previously published flux [Adriani et al., 2011].

\subsection{Systematic uncertainties}

Systematic uncertainties include the statistical errors resulting from the finite size of the efficiency samples, the Live Time uncertainty, the uncertainty due to the unfolding procedure and the error associated to the flux normalization factors. Figure 3 shows the time dependence of the systematic uncertainties as a function of the rigidity.

\section{Conclusions}

The time dependence of the helium fluxes measured by PAMELA offers the possibility to study the propagation mechanism of GCRs inside the heliosphere. Finally, by comparing modulation of helium and modulation of protons is possible to evaluate difference in LIS shape and possible different amount of modulation between the two species due to dependencies on particle's charge or mass. The helium fluxes have been computed for time periods of one Carrington Rotation between 
July 2006 and December 2009, in the energy range from $80 \mathrm{MeV} / n$ and $\sim 20 \mathrm{GeV} / n$; preliminary results will be presented at the conference and they will be the topic of an upcoming paper.

\section{References}

[D’Agostini, 1995] D’Agostini, G. (1995), A multidimensional unfolding method based on Bayes theorem., Nuclear Instruments and Methods in Physics Research A, 362(2-3):487-498.

[Adriani et al., 2011] PAMELA measurements of cosmic-ray proton and helium spectra., Science, 332:69-71.

[Munini et al., 2019] Isotope solar modulation with the PAMELA experiment., This Conference Proceeding of Science, 2019.

[Adriani et al.] Adriani, O., Bonechi, L., Bongi, M., et al. 2003, Nuclear Instruments and Methods in Physics Research A, 511, 72

[Adriani et al.(2013)] Adriani, O., Barbarino, G. C., Bazilevskaya, G. A., et al. 2013, ApJ, 765, 91

[Adriani et al.(2014)] Adriani, O., Barbarino, G. C., Bazilevskaya, G. A., et al. 2014, Phys. Rep., 544, 323

[Adriani et al.(2015)] —. 2015, ApJ, 810, 142

[Adriani et al.(2016)] —. 2016, Physical Review Letters, 116, 241105

[Aslam \& Badruddin(2015)] Aslam, O. P. M., \& Badruddin. 2015, Solar Phys., 290, 2333

[Boezio et al.(2002)] Boezio, M., Bonvicini, V., Mocchiutti, E., et al. 2002, Nuclear Instruments and Methods in Physics Research A, 487, 407

[Jiang et al.(2015)] Jiang, J., Cameron, R. H., \& Schüssler, M. 2015, Astrophys. J. Lett., 808, L28

[Mewaldt et al.(2010)] Mewaldt, R. A., Davis, A. J., Lave, K. A., et al. 2010, Astrophys. J. Lett., 723, L1

[Orsi et al.(2005)] Orsi, S., Carlson, P., Hofverberg, P., Lund, J., \& Pearce, M. 2005, International Cosmic Ray Conference, 3, 369

[Osteria et al.(2004)] Osteria, G., Campana, D., Barbarino, G., et al. 2004, Nuclear Instruments and Methods in Physics Research A, 535, 152

[Picozza et al.(2007)] Picozza, P., Galper, A. M., Castellini, G., et al. 2007, Astroparticle Physics, 27, 296

[Potgieter(2013)] Potgieter, M. S. 2013, Space Sci. Rev., 176, 165

[Schröder et al.(2017)] Schröder, K.-P., Mittag, M., Schmitt, J. H. M. M., et al. 2017, , 470, 276

[Stozhkov et al.(2005)] Stozhkov, Y. I., Basili, A., Bencardino, R., et al. 2005, International Journal of Modern Physics A, 20, 6745

[Strauss \& Potgieter(2014)] Strauss, R. D., \& Potgieter, M. S. 2014, Advances in Space Research, 53, 1015 